\title{
Protée
}

\section{Les pierres du temps}

Archéologie de la nature, géologie de la ruine

\section{Michel Makarius}

Volume 35, numéro 2, automne 2007

Imaginaire des ruines

URI : https://id.erudit.org/iderudit/017469ar

DOI : https://doi.org/10.7202/017469ar

Aller au sommaire du numéro

\section{Éditeur(s)}

Département des arts et lettres - Université du Québec à Chicoutimi

ISSN

0300-3523 (imprimé)

1708-2307 (numérique)

Découvrir la revue

Citer cet article

Makarius, M. (2007). Les pierres du temps : archéologie de la nature, géologie de la ruine. Protée, 35(2), 75-80. https://doi.org/10.7202/017469ar
Résumé de l'article

"C'est tout l'attrait des ruines de permettre qu'une oeuvre humaine soit presque perçue comme un produit de la nature ». Tout en confirmant cette thèse de Simmel, le premier Romantisme (Novalis, Schiller) développe une " rêverie minéralogique » (Breton) où nature et culture échangent leurs signes : l'art est apprécié pour son matériau naturel tandis que les formes de la nature sont envisagées sous leur aspect esthétique. En interrogeant cette rêverie dans le cadre de la modernité du Land Art de Robert Smithson, nous voyons comment, là encore, la proposition de Simmel s'inverse de telle sorte que c'est tout l'attrait des ruines de permettre qu'une oeuvre de la nature soit presque perçue comme un produit de l'art. 


\title{
LES PIERRES DU TEMPS ARCHÉOlOGIE DE LA NATURE, GÉOLOGIE DE LA RUINE
}

MICHEL MAKARIUS

\author{
Tu as plus de chance, Amérique \\ Que n'en a le vieux continent, \\ Tu n'as ni châteaux en ruine \\ Ni roches basaltiques.
}

Goethe (1979-1982: 721)

Entre la première Renaissance et le $\mathrm{XVI} \mathrm{I}^{\mathrm{e}}$ siècle, la signification des ruines change complètement: de rappel de la culture gréco-latine qu'elles étaient d'abord, les ruines deviennent le modèle d'une forme en perpétuelle métamorphose. Se faisant l'écho du recyclage des "monceaux pierreux" qui jonchent la ville éternelle en de nouveaux matériaux de construction, Joachim du Bellay évoque, dans les Antiquités de Rome, le spectacle quotidien de la récupération des restes de la cité antique. S'il critique les ambitions urbaines du pouvoir pontifical, le poète ne cache pas sa fascination pour l'énergie d'une ville en continuelle reconstruction. Comme l'écrit Michel Jeanneret:

L'attrait des profondeurs et des zones vagues traverse le recueil, ainsi qu'en témoigne un réseau lexical qui s'organise autour d'images comme poudre, ombre, ruine, reliques, monceaux, nue, vapeur et fait rimer poudreux, cendreux, ténébreux et ombreux. [...] Dans la méditation sur le travail de l'art s'infiltre, avec cette image d'un surgissement à partir des racines profondes, le modèle de la reproduction biologique. Un scénario désormais familier se confirme. (1977: 104)

L'esthétique maniériste se reconnaîtra dans ce principe germinatif de la matière chaotique et primitive que Léonard avait déjà exprimé dans ses dessins. À l'image du Forum romain envahi par une végétation prolixe et anarchique, le désordre des vestiges antiques, que se disputent herbes et ronces, apparait comme la symbiose spontanée de la nature et de la culture. Symbiose qui culminera à la fin du XVI ${ }^{\mathrm{e}}$ siècle, dans l'aménagement des fontaines et des fausses grottes des jardins des villas patriciennes. Avec leurs parois revêtues d'authentiques concrétions minérales (roches volcaniques, coquillages, corail, nacre, etc.), ces lieux renvoient de manière 
fantasmatique aux spéculations sur l'origine et la formation des minéraux. Dans les salles ombreuses et humides de la grotte des jardins de Boboli à Florence se joue ainsi une savante cosmogonie; les parois tapissées de croûtes calcaires et de reliefs fossiles racontent des histoires de boues mêlées, d'eau pétrifiée, de ruissellement ou de Déluge, qu'une natura ludens se plaît à signer. Comme le jardin fait référence au Paradis perdu, la grotte évoque le ventre fécond de la Mère Nature, source de toute vie. Dans ces lieux où «la nature imite l'art qui la prend pour modèle» (Morel, 1998: 91), la fascination maniériste pour la réversibilité des signes est à son comble. Autant qu'il souhaite égarer le promeneur dans les vertiges d'une mimesis à tiroir, le microcosme de la grotte se veut l'origine secrète du jardin, son humus primitif où, dans l'indétermination des éléments en gestation, la matière prend forme avant même de se scinder en culture et nature. Ces retraites enchantées invitent à des rêveries qui penchent naturellement vers les récits mythologiques consignés dans les Métamorphoses d'Ovide. Pas de fantaisie minéralogique qui ne soit aussi une interrogation passionnée sur la Genèse. «Le temps, ce grand sculpteur» ${ }^{1}$, est un artiste mystérieux.

Avant d'être une méditation sur l'Histoire et la destinée humaine, la poétique des ruines appartient donc à la lithologie dont elle ne se détachera jamais complètement. En perpétuant la tradition marquée par les noms de Paracelse, de Bohme et de Kircher pour lesquels le dessin des pierres imagées est dû à la main d'une Nature artiste (Baltrusaitis, 1995) -, la Naturphilosophie, cette science romantique, cherchera, à son tour, à déchiffrer les secrets des minéraux. Dans la mesure où son projet est de découvrir l'unité de l'Univers, il n'est pas question de séparer sujet et objet, pensée et matière, produit de l'Histoire et règne de la Nature. Bien au contraire: une secrète sympathie circule entre les deux domaines, si bien que science et art ne cessent de communiquer. Schiller écrira en 1786:

Une nouvelle expérience dans cet empire de la vérité autrement dit les sciences naturelles -, la gravitation, la découverte de la circulation sanguine, le système de la Nature de
Linné ont au fond, pour moi, la même signification qu'une antiquité découverte à Herculanum - ce sont toujours le reflet d'un esprit et ils permettent chacun la connaissance d'un être semblable. (Cité dans Le Blanc et alii, 2003: 335)

Il n'est pas jusqu'à Kant qui n'envisage de retracer le lien organique qui relie entre elles les différentes disciplines des sciences naturelles, si bien que la Critique de la faculté de juger nomme «archéologue de la Nature» celui qui a vocation de reconstituer la chaîne qui remonte à «la mère universelle».

C'est ainsi que les artistes, les écrivains et les peintres romantiques retrouveront dans les ruines ce que la forme et l'usage avaient fini par occulter, à savoir leur qualité physique qui les rattache originairement au monde minéral. Si l'on interprète habituellement leur inclination pour la période gothique en fonction de sa signification idéologique et esthétique, il faut également prendre la mesure de ce qu'il convient d'appeler une lithophilie ou une lithomanie à l'endroit de leur imaginaire des ruines. Et l'on doit insister sur le rôle que tiennent les sciences de la nature dans la formation et dans l'inspiration de la génération du premier romantisme (Schefer, 2006: 88). On sait qu'Heinrich Schubert était médecin, naturaliste et philosophe, Johan Ritter, philosophe et physicien, Novalis, poète, mathématicien et ingénieur. Il est particulièrement remarquable qu'au sein de ces sciences, la géologie (ou plutôt ce que l'on appelait à la fin du XVIII e siècle la "géognose») tient une place stratégique. Certains s'y étaient initiés pour des raisons professionnelles: après un passage à l'Académie des Mines de Freiberg (1798-99), Novalis sera nommé assesseur des salines de Weissenfels en Thuringe; avant lui, Gothe avait été chargé de superviser la remise en état de la mine de Ilmenau au sud-ouest de Weimar. D'autres y sont attirés par un mélange de curiosité scientifique et d'attrait esthétique que l'on retrouve dans le débat sur la représentation picturale du paysage. Gustav Carus, obstétricien réputé avant d'être peintre et ami de Friedrich, ajoutera à ses Lettres sur la peinture de paysage une brève Physiognomonie des montagnes. 
Pour ces arpenteurs infatigables, ramasseurs impénitents de cailloux, la science des pierres et des gemmes n'est pas une discipline vouée à de sèches classifications. Bien au contraire, l'étude du monde non organique, de sa morphologie et de sa composition, alimente une invraisemblable «minéralogie visionnaire" pour reprendre l'expression d'André Breton. Sans entrer dans la querelle entre neptuniens et plutoniens (ou "vulcanistes"), pour lesquels l'origine de la Terre remonte, respectivement, soit à une immersion totale, soit à une explosion interne, la minéralogie est ici l'étude des formes concrètes du temps. Les processus de sédimentation et le mystère des pierres fossiles orientent ainsi la spéculation vers la quête de l'origine. Dans le récit de son expédition sur l'île de Rügen, qu'il effectue en suivant les traces de Caspar Friedrich qui en a peint les célèbres falaises, Gustav Carus mêle l'évocation mythologique à la description géologique:

Le sable était truffé de rognons de silex isolés et partiellement

réduits en fragments qui eux aussi, avec plus ou moins

d'évidence, s'avéraient être des concrétions fossilisées, si bien que par delà les beautés de la forme en ses lignes et couleurs, mon regard plongeait plus au cour de l'objet, d'une vision plus lucide, dans maints mystères de la science! [...] Je n'ai jamais été, autant qu'alors, en cet endroit, en aussi intime contact avec le vieux monde légendaire de notre souche nordique. (1999: 13)

Le regard sur les pierres confond ainsi les temps immémoriaux de la Création avec le passé de l'humanité, au risque de fantasmer une fusion archaïque du sol et du sang. Novalis, de son côté, accomplit le chemin inverse en allant de l'œuvre d'art, la sculpture, à son matériau brut, la pierre: «ce n'est qu'en ces statues qui nous restent des temps passés de la beauté humaine», écrit-il dans le conte initiatique Les disciples à Saïs, "que transparaissent ainsi l'esprit profond et la compréhension singulière du monde minéral»(2004: 106). Même attitude chez Gœethe lors de son voyage en Italie en 1787, lorsqu'il s'intéresse davantage à la qualité du granit des obélisques égyptiens qu'à leur valeur d'artefact (Lacoste, 1999).
L'approche des vestiges antiques par le biais de la lithologie corrobore donc la remarque de Georg Simmel selon laquelle "c'est tout l'attrait des ruines de permettre qu'une œuvre humaine soit presque perçue comme un produit de la nature» (1998: 113). Dans la peinture romantique des ruines, le paysage et l'homme, le vivant et l'inerte, le naturel et l'artificiel, plutôt que de s'opposer, se rejoignent. Turner greffe des arabesques de lierre sur les arches et les ogives de ses abbayes gothiques. Karl Blechen enracine les piliers de sa cathédrale dans un sol moussu (Église gothique en ruine, 1826). Caspar Friedrich dresse une abside ajourée entre des arbres désespérément émondés (L'Abbaye dans une forêt de chênes, 1809); transforme la forêt en sanctuaire où surgit une cathédrale parmi les sapins (Crucifix dans la montagne, 1812); fait d'une clairière une nef (Les Ruines d'Eldena, 1824)². Chaque fois, les tableaux montrent une végétation reprenant dans son giron le vestige architectural telle une mère retrouve son enfant perdu; comme si, au fond, la construction d'un édifice n'avait été qu'un moment d'égarement, une fantaisie de la nature qui, par le truchement de sa créature pensante, l'homme, s'était laissé aller à une étrange excroissance. À la suite de Gothe, et parfois contre lui, les peintres (Friedrich, Carus, Schinkel, Runge) de même que les écrivains (les frères Schlegel et Novalis) envisagent ainsi la nature comme l'expression de la toute-puissance de Dieu; son spectacle transporte l'homme hors de luimême en lui faisant prendre conscience de «l'unité dans l'infinité de l'univers" (Carus et Friedrich, 1988: 68). Chaque brin d'herbe parcouru par un souffle divin sacralise le paysage et fait du tableau une image pieuse, tandis qu'à leur tour les éléments du paysage prennent l'aspect d'un artefact. À travers les configurations inorganiques du monde minéral, nature et culture échangent leur signe: l'art est apprécié pour son matériau naturel tandis que les configurations naturelles sont envisagées sous leur forme esthétique. D'où la possibilité de renverser ainsi la proposition de Simmel: c'est tout l'attrait des ruines de permettre qu'une oeuvre de la nature soit presque perçue comme un produit de l'art. 
Franchissons maintenant deux siècles et un océan pour atterrir sur le continent américain, plus précisément dans l'Utah, sur le Lac salé où Robert Smithson a construit sa fameuse Spiral Jetty. Aussi loin que nous puissions être dans le temps et dans l'espace des paysages mélancoliques de Caspar Friedrich, il est difficile de ne pas voir dans cette œuvre emblématique du Land Art, comme dans les textes de Smithson, une problématique qui renoue avec les Romantiques. On a déjà relevé que le Land Art, par la démesure des espaces qu'il embrasse, est l'expression contemporaine du sublime, «ce par rapport à quoi tout le reste est petit» disait Kant. Mais le rapport entre Smithson et les Romantiques passe encore par d'autres chemins; à commencer par la même «lithophilie» qui le conduit à une "chasse aux minéraux» dans le New Jersey et que raconte son texte Le Pays $d u$ cristal $^{3}$. Comme pour les Romantiques, les pierres conçues comme des morceaux de l'infini spatial et temporel suscitent ici aussi une vaste rêverie: chez les auteurs romantiques, la ruine et le "bloc erratique" projettent leur ombre jusque sur l'écriture en fragment ${ }^{4}$. Chez Smithson, la «géognose» spécule sur l'analogie avec le langage. Ainsi on peut lire dans «Une sédimentation de l'esprit: Earth Projects»:

Le nom des minéraux et les minéraux eux-mêmes ne diffèrent pas les uns des autres, parce qu'au fond des deux - matériau et chose imprimée - commence un nombre abyssal de fissures. Les mots et les roches contiennent un langage qui suit une syntaxe de fentes et de ruptures. Il suffit de regarder n'importe quel mot assez longtemps pour le voir s'ouvrir en une série de failles, en un terrain de particules, chacune d'entre elles contenant son propre vide. (1994: 195)

Étonnante linguistique qui indexe le langage sur un chaos originel comme si la Terre et l'esprit étaient issus d'un même magma! On ne s'étonnera pas de trouver, parmi les papiers de Smithson, un croquis fait d'après une gravure de Kircher illustrant l'origine des montagnes et des volcans par des fleuves de feu souterrains traversant le globe à partir d'un noyau incandescent. Cette cosmogonie baroque et débridée voulait déchiffrer le Livre de la Nature par-delà la multiplicité, la diversité et l'irrégularité de ses manifestations. Après avoir fasciné les penseurs de la Naturphilosophie, cette théorie ne pouvait qu'intéresser Smithson. Sa minéralogie visionnaire, qui n'a rien à envier à celle des Romantiques, débouche ainsi sur une pensée singulière du temps et de l'espace qu'il désigne par le terme d'entropie. Smithson rappelle que cette notion, qui provient de la physique, désigne une perte d'énergie; la reprenant à son compte, il désigne ici par entropie le paysage (ou le territoire) qui apparaît comme une sorte de trou noir où le temps absorberait l'espace. À la suite d'une randonnée dans la zone d'un terrain vague de Passaic dans le New Jersey, Smithson raconte:

[...] ce panorama zéro paraissait contenir des ruines à l'envers, c'està-dire toutes les constructions qui finiraient par y être édifiées. C'est le contraire de la «ruine romantique», parce que les édifices ne tombent pas en ruines après qu'ils aient été construits, mais qu'ils s'élèvent en ruines avant même de l'être.

L'architecture est ainsi expression même de l'entropie: «au lieu de nous remémorer le passé comme le font les monuments anciens, les nouveaux semblent faire oublier l'avenir» (ibid.: 162), dit encore Robert Smithson. Outre l'architecture, Smithson donne d'autres cas d'entropie: un black out accidentel, un tremblement de terre, une éruption volcanique, mais aussi l'affaire du Watergate ou encore une séance de cinéma qu'il définit comme «un trou dans sa propre vie». Ces exemples, qui mêlent histoire et nature, expriment tous la même chose: un état de crise où l'espace et le temps s'annulent, où les énergies libérées atteignent un point d'inertie, un vanishing point. En diffèrent seulement la vitesse et l'intensité.

À l'instar des déserts californiens dont Baudrillard disait qu'ils «vous entraînent dans le vertige du temps, dans l'éternité minutieuse d'une catastrophe au ralenti» (2005: 9), la Spiral Jetty figure l'enroulement du temps dans l'espace. Signes purs de l'infini, les cristaux de sel du lac de l'Utah retiennent prisonniers les temps préhistoriques dont les engins de 
remblaiement sont les nouveaux dinosaures. Après «la tragédie du paysage» peinte par Friedrich selon la fameuse formule de David d'Angers, voici maintenant le degré zéro du chaos.

[...] tandis que je regardais le site, il se réverbéra sur tout

l'horizon comme pour suggérer un cyclone immobile, pendant que le vacillement de la lumière faisait trembler le panorama tout entier. Une sorte de secousse assoupie se répandit dans l'immobilité palpitante, en une sensation de tournoiement sans mouvement. Ce site était un rond-point qui s'enfermait dans une immense rondeur. De cet espace en giration, surgit la virtualité de la Spiral Jetty. [...] Ce fut comme si une succession d'ondes et de pulsations faisaient osciller la terre ferme et que le lac demeurait tranquille comme un roc. La bordure du lac devint la bordure du soleil, une courbe bouillante, une explosion se soulevant en une protubérance enflammée. La matière s'effondra dans le lac, s'y reflétant en forme de spirale.

(Smithson, 1994: 206)

On le sait, de nombreuses créations du Land Art s'inscrivent dans de tels espaces de sidération. Reflet en creux du paysage européen modelé par l'histoire, l'entropie relève du territoire américain dont l'immensité géographique tient lieu de profondeur historique, comme l'exprime de manière emblématique La Conquête de l'Ouest. Mais il convient de souligner l'ambivalence de ces œuvres monumentales; par sa situation et son échelle, l'intervention humaine imite une création naturelle tandis que, par sa forme régulière et géométrique, elle signale une forme artificielle. Telle est son utopie au sens le plus littéral puisque nature et culture se réconcilient dans un site hors de portée, un non-lieu: utopos.
C'est dire que l'imaginaire ruiniste du Nouveau Monde (au moins jusqu'à la date du 11 septembre 2001) explore le retrait $d u$ temps plus que sa lancinante permanence comme le veut la tradition européenne. L'attraction récurrente de Smithson pour les miroirs (Map on mirror, Mirror vortex, Mirror displacement, etc.) et l'emploi de matériaux denses pénétrés de forces chtoniennes sont les deux faces d'une même médaille. La surface lisse et froide du pan miroirique et la substance épaisse des coulées de terre, de plomb ou d'asphalte - aussi antinomiques soient-elles expriment chacune un temps absent. La première parce que, comme l'attestent les façades des architectures de verre et d'acier, le temps n'y a pas de prise. La seconde parce que, à l'inverse, la masse inerte de la nature primitive le retient enfoui. À de nombreuses occasions (Mirror displacement), l'artiste fait se rencontrer les deux éléments pour révéler leur secrète réversibilité; le réel et son double, l'image et son modèle, la surface réfléchissante et le bloc compact, le brillant et le mat sont, l'un de l'autre, le passé et le futur.

La disparition du temps dans le trou noir de l'entropie se nomme plus simplement catastrophe. Dans l'irruption soudaine de l'événement inouï, le temps se fige tel un arrêt sur image (sur le modèle du 11 septembre 2001, qu'une date suffit à désigner). Hypostase de la nature et de la culture, la catastrophe devient alors le comble de la ruine contemporaine. $\mathrm{Si}$, à l'époque romantique, elle appartenait aux forces du sublime, elle est aujourd'hui l'horizon désenchanté de notre paysage historique et naturel: à l'infini du ciel étoilé de Kant se substitue désormais l'implacable planité d'un Grand Lac Salé. 


\section{NOTES}

1. Voir Les Voix intérieures de V. Hugo (1837).

2. On pourrait d'ailleurs faire toute une typologie du motif de l'arbre chez Friedrich en montrant sa situation quasi ontologique: tantôt il se détache au premier plan et se dresse solitaire face à la plaine à l'instar des personnages vus de dos; tantôt un groupe clairsemé de troncs aux branches hurlantes dialogue avec les restes d'une abbaye.

3. Reproduit dans Smithson (1994: 169-170).

4. Voir ce fragment de Novalis: «Ressemblance de la géognosie

historique et de l'oryctonosie avec la philologie" (2004 : fragment 470; 244).

\section{RÉFÉRENCES BIBLIOGRAPHIQUES}

BALTRUSAITIS, J. [1995]: Aberrations - Les perspectives dépravées 1, Paris, Flammarion, coll. "champs".

Baudrillard, J. [(1986) 2005]: Amérique, Paris, Le livre de poche. CARUs, G. C. [1999]: Voyage à l'île de Rügen, trad. N. Taubes, SaintMaurice, Premières Pierres.

CARUS, G. C. et C. D. Friedrich [1988]: De la peinture de paysage dans l'Allemagne romantique, trad. par E. Dickenherr, A. Pernet et R. Rochlitz, Paris, Klincksieck.

G(ethe, J. W. [1979-1982]: Poésies, trad. de R. Ayrault, vol. 2, Paris, Aubier.

Hugo, V. [(1837) 1985]: Les Voix intérieures, CEuvres complètes. Poésie I, Paris, Laffont, coll. "Bouquins".

JeAnneret, M. [1997]: Perpetum mobile. Métamorphoses du corps et des œuvres de Vinci à Montaigne, Paris, Macula.

KANT, E. [1965]: Critique de la faculté de juger, Paris, Vrin. Lacoste, J. [1999]: Le "Voyage en Italie» de Gothe, Paris, PUF.

Le Blanc, C., L. Margantin et O. Schefer [2003] : La Forme poétique du monde. Anthologie du romantisme allemand, Paris, Corti. MOREL, P. [1998]: Les Grottes maniéristes, Paris, Macula. NOvalis [2004]: Semences, trad. O. Schefer, Paris, Allia. SCHEFER, O. [2006] : Résonances du romantisme, Bruxelles, La lettre volée.

Simmel, G. [1998]: «Les ruines. Un essai d'esthétique», La Parure et autres essais, Paris, Maison des sciences de l'homme, 111-118.

SMITHSON, R. [1994]: Une rétrospective : le paysage entropique, 1960/1973 (catalogue d'exposition), Marseille, Musée de Marseille/Réunion des musées nationaux. 